\title{
Produtos naturais de algas marinhas e seu potencial antioxidante
}

\author{
Fabíola Dutra Rocha ${ }^{1,2}$, Renato Crespo Pereira ${ }^{3}$, Maria Auxiliadora Coelho Kaplan ${ }^{1}$, \\ Valéria Laneuville Teixeira ${ }^{3 *}$
}

\author{
${ }^{1}$ Núcleo de Pesquisa de Produtos Naturais, Universidade Federal do Rio de Janeiro, Ilha do Fundão, 21941-590, \\ Rio de Janeiro, RJ, Brasil, \\ ${ }^{2}$ NUPESC, Faculdade e Fundação UNIRG, Alameda Madrid 545, Jardim Sevilha, 77410-470, Gurupi, TO, \\ Brasil, \\ ${ }^{3}$ Departamento de Biologia Marinha, Instituto de Biologia, Universidade Federal Fluminense, \\ P.O. Box 100.644, 24001-970, Niterói, RJ, Brasil
}

\begin{abstract}
RESUMO: Os radicais livres e outros derivados ativos do oxigênio são inevitavelmente coproduzidos em algumas reações biológicas e exercem papel fisiológico importante. No entanto, essas espécies reativas têm sido descritas como fatores que participam diretamente dos mecanismos fisiopatológicos relacionados com a continuidade e as complicações de diversos estados patológicos como a aterosclerose, a diabetes, o câncer, a artrite reumatóide, entre outros. Dessa forma, a terapia antioxidante preveniria o desenvolvimento e a progressão dessas complicações. As algas marinhas representam uma importante fonte de substâncias antioxidantes naturais, uma vez que têm sistemas de defesas antioxidantes bem desenvolvidos. O presente trabalho é uma compilação das pesquisas realizadas sobre a atividade antioxidante de produtos naturais marinhos e extratos de algas marinhas bentônicas.
\end{abstract}

Unitermos: Estresse oxidativo, antioxidantes, algas marinhas bentônicas.

\begin{abstract}
Natural products from marine seaweeds and their antioxidant potential" Free radicals and other reactive species of oxygen are co-produced in some biological reactions and they play important physiological role, and nevertheless they are reported as factors that take straight part in the pathophysiologic mechanism associated with continuity and complications of the several pathological process such as arteriosclerosis, diabetes mellitus, cancer, and arthritis, among others. In this way, the antioxidant therapy should prevent the development and progress of these complications. Seaweeds can be valuable source of natural antioxidant compounds since they have a well-developed antioxidant defense system. The present work is a compilation of the antioxidant activities of marine natural products and benthonic marine seaweeds crude extracts researches.
\end{abstract}

Keywords: Oxidative stress, antioxidants, benthonic marine seaweeds.

\section{INTRODUÇ̃̃O}

$\mathrm{O}_{2}$ tem um significado fundamental para os organismos aeróbios, pois participa na obtenção de energia na forma de ATP, através da cadeia respiratória, como aceptor final de elétrons. Participa também de várias reações metabólicas como a biossíntese de prostaglandinas e esteróides e na oxidação de muitas substâncias aromáticas, entre outras (Fleschin et al., 2000). Os radicais livres e outros derivados ativos do oxigênio são inevitavelmente co-produzidos nessas reações biológicas e exercem papel fisiológico importante, mas também estão envolvidos em vários processos deletérios ao organismo humano como o câncer, a aterosclerose, a Diabetes mellitus, a artrite reumatóide, a distrofia muscular, a catarata, as desordens neurológicas e o processo de envelhecimento (Nordberg; Arnér, 2001). Finalmente, a presença de radicais livres tem sido correlacionada com um grande número de doenças, mas não como agentes etiológicos e sim como fatores que participam diretamente dos mecanismos fisiopatológicos, os quais determinam a continuidade e as complicações de diversos estados patológicos (Rover Júnior et al., 2001).

Os organismos aeróbios, desde as cianobactérias até o homem, desenvolveram uma série de mecanismos fisiológicos e biomoleculares de defesa contra os efeitos das espécies reativas do oxigênio (EROs). Nas células de organismos fotossintetizantes esses mecanismos estão mais fortemente desenvolvidos em comparação com outras células, uma vez que as membranas fotossintéticas, os tilacóides, são alvo primário para os efeitos deletérios oxidativos, por conterem lipídeos não saturados como elementos estruturais majoritários. Portanto, vários mecanismos de proteção são desenvolvidos nessas células. De fato, as folhas 
das plantas vasculares e os talos das algas contêm numerosas substâncias antioxidantes. As algas estão sempre submetidas a rápidas variações de intensidade de luz e concentrações de $\mathrm{O}_{2}$ e $\mathrm{CO}_{2}$ ao longo da coluna de água e, assim, sua sobrevivência depende de uma resposta eficiente ao estresse oxidativo. Por essa razão, esses organismos podem representar uma importante fonte de substâncias antioxidantes naturais tanto para as indústrias alimentícias como para as farmacêuticas (Matsukawa et al., 1997).

A definição mais aceita para antioxidantes (AO) é que seriam substâncias as quais, mesmo presentes em baixas concentrações em relação ao substrato oxidante, poderiam atrasar ou inibir as taxas de oxidação (Sies, 1993). Vários componentes fisiológicos são conhecidos pela capacidade de inativar ou neutralizar radicais livres, como o $\alpha$-tocoferol, o ascorbato, o $\beta$-caroteno, o ácido úrico, a ubiquinona, a transferrina, a ceruloplasmina, entre outros (Olszewer, 1995; Fleschin, 2000; Matkovics, 2003). Alguns metais são cofatores na síntese de radicais livres e, substâncias quelantes de metais como o EDTA, a desferroxamina e a D-penicilamina são empregados como antioxidantes exógenos (Olszewer, 1995; Olszewer; Carter, 1988; Tully, 1999). Substâncias naturais com atividade redutora e neutralizadora de radicais livres são muito usadas como agentes antioxidantes exógenos. (Favier et al., 1995; Rice-Evans et al., 1996; Pietta, 2000; Nordberg; Arnér, 2001). De fato, os produtos naturais parecem ser uma fonte promissora de substâncias com atividade antioxidante e pesquisas realizadas nos últimos anos evidenciam que o enriquecimento dos sistemas orgânicos com antioxidantes naturais pode corrigir a homeostasia viciada (Tiwari, 2001).

Constam na literatura vários trabalhos de busca de substâncias com atividade antioxidante (AAO) em algas (Tabela 1), porém, no Brasil, esse campo de pesquisa ainda não foi devidamente explorado, apesar da riqueza da nossa flora ficológica marinha.

\section{ALGAS MARINHAS E SEU POTENCIAL ANTIOXIDANTE}

$O$ interesse inicial pelo estudo de substâncias com atividade antioxidante (AAO) em algas surgiu no Japão, na busca de novos aditivos para alimentos, em substituição àqueles antioxidantes sintéticos utilizados, como o hidrixianisol butilado (BHA) e o hidroxitolueno butilado (BHT), os quais mostravam efeitos carcinogênicos, alterações enzimáticas e lipídicas em animais. O fato de algumas algas secas poderem ser estocadas por um longo período sem o perigo de deterioração oxidativa, mesmo apresentando mais de $30 \%$ do total de seus ácidos graxos na forma de cadeias poliinsaturadas (principalmente as algas pardas), despertou o interesse dos pesquisadores em relação ao mecanismo $\mathrm{AO}$ presente nessas algas
(Fujimoto; Kaneda, 1980). Fujimoto e Kaneda (1980) usaram o teste de estabilidade do éster metílico do óleo de girassol e constataram que $60 \%$ das frações solúveis em $\mathrm{CHCl}_{3}$ dos extratos das algas testadas foram capazes de estender o período de indução da reação do substrato éster (tempo necessário para atingir um ponto crítico de oxidação). As algas pardas, em geral, apresentaram maior atividade antioxidante, particularmente, Eisenia byciclis, Sargassum kjellmanianum, Ishige okamurae, Undaria pinnatifida, Heterochordaria abientina e Scytosiphon lomentaria. A partir do fracionamento dos extratos em $\mathrm{CHCl}_{3}$ de E. byciclis e U. pinnatifida, os autores identificaram como princípios ativos responsáveis pela AAO as frações fosfolipídicas (fosfatidiletanolamina e fosfatidilinositídeo) e o carotenóide fucoxantina, respectivamente. Em um outro trabalho os mesmos autores pesquisaram a AAO de 36 espécies de algas bentônicas e, novamente, constataram que as algas pardas apresentavam maior AAO (Fujimoto; Kaneda, 1984).

Em 1990, Le Tutour et al. (1990) pesquisou a AAO dos extratos apolares de 7 algas marinhas bentônicas da costa francesa. Mais uma vez os resultados para as algas pardas foram os melhores, principalmente os obtidos com Laminaria digitata e Himanthalia elongata. Os extratos dessas duas espécies mostraram sinergia com a vitamina $\mathrm{E}$ no teste de estabilidade do éster metílico do óleo de girassol. Cahyana et al. (1992), identificaram a pirofeofitina a , um metabólito da clorofila a, como um dos princípios antioxidantes presente no extrato da alga Eisenia bicyclis, alga parda comestível muito popular no Japão. Em um trabalho posterior, Cahyana et al. (1993), analisaram os possíveis efeitos sinérgicos de derivados porfirínicos (clorofila a , feofitina a e pirofeofitina a) com o $\alpha$-tocoferol e ácido ascórbico, através dos métodos do tiocinato e substâncias reativas ao ácido tiobarbitúrico. Todas as substâncias testadas mostraram melhor efeito $\mathrm{AO}$ quando na presença de $\alpha$-tocoferol ou ácido ascórbico, sugerindo um efeito sinérgico.

Foti et al. (1994), sabendo que meroditerpenos (Figura 1: estruturas 1-2) isolados de algas pardas do mar Mediterrâneo do gênero Cystoseira são análogos aos tocoferóis e, portanto, com potencial para uma boa AAO, como aromaticidade e alto nível de oxigenação, fizeram uma avaliação das propriedades dessas substâncias como quelantes de ${ }^{1} \mathrm{O}_{2}$ (através do teste de decomposição térmica do 1,4-dimetilnaftaleno 1,4-endoperóxido, em comparação com o padrão 2,5-difenil-3,4-benzofurano), ânion superóxido (ensaio da xantina-oxidase) e radicais peróxidos (ensaio da inibição da peroxidação do -caroteno, mediada pelo radical peróxido do linoleato de metila na presença de $\mathrm{O}_{2}$ e radical iniciador 2,2'-azobis-(2-metilpropionitrila) - AIBN). Os três metabolitos testados apresentaram boa atividade sequestrante do ${ }^{1} \mathrm{O}_{2}$ e nenhuma atividade sobre o ânion superóxido. As substâncias 1 e 2 também foram 
ativas contra os radicais lipoperóxidos.

Os "florotaninos" formam uma classe de substâncias naturais características de Phaeophyceae e de grande potencial farmacológico. Essas substâncias caracterizam-se por uma unidade estrutural de floroglucinol (1,3,5-triidroxibenzeno) e por terem as características químicas dos taninos, e.g., estruturas 3 a 7 (Figura 1). Em 1996, Yan et al. (1996) avaliaram a AAO dos florotaninos de Sargassum kjellmanianum, usando o ensaio da determinação do período de indução da oxidação dos ácidos graxos (tempo necessário para se atingir um ponto crítico de oxidação do óleo de peixe acarretando um aumento na massa total dos ácidos graxos em 6\%). Os autores constataram que os florotaninos têm uma boa AAO contra a rancificação do óleo de peixe, sendo inclusive cerca de 2,6 vezes maior do que o BHT. $\mathrm{O}$ método do tiocianato foi usado por Anggadiredja et al. (1997) para avaliar a AAO dos extratos em metanol, em éter etílico e em hexano das algas, secas e frescas, Sargassum polycystum (alga parda) e Laurencia obtusa (alga vermelha), coletadas nas águas das Ilhas Seribu (Indonésia). Os resultados revelaram que os extratos das algas secas não apresentaram atividade antioxidante, enquanto que os extratos das algas frescas foram muito ativos. Para $S$. polycystum o extrato metanólico mostrou melhor atividade do que aqueles em éter etílico e em hexano. Ainda, em 1997, Matsukawa et al. (1997) avaliaram os extratos aquosos e etanólicos de 17 espécies de algas quanto a AAO por inibição da enzima lipoxigenase e atividade redutora de radical livre (RL) pelo ensaio de descoloração do 1,1-difenil2-picrilidrazila (DPPH). Os resultados mostraram que os extratos em etanol possuem AAO, sendo que os extratos das algas pardas, nos dois ensaios, mostraram os melhores resultados. Nesse estudo, as 3 espécies de Sargassum avaliadas mostraram melhor atividade do que os demais grupos de algas testadas. O extrato da alga Eisenia bicyclis também mostrou atividade nos dois ensaios (inibição da lipoxigenase e atividade redutora do radical DPPH), corroborando os resultados de Fujimoto e Kaneda (1980). Nenhuma correlação entre os mecanismos de inibição enzimática e atividade neutralizadora de radicais foi observada.

Em 1998, Le Tutour (1998) ampliou o trabalho de 1990, pesquisando os extratos de mais três algas pardas: Fucus vesiculosus, F. serratus e Ascophyllum nodosum com o intuito de isolar e identificar os princípios responsáveis pela AAO e verificar a sinergia com a vitamina $\mathrm{E}$. Todos os extratos testados mostraram a propriedade de estender o período de indução da oxidação do substrato e efeito sinérgico com a vitamina E. Na purificação dos extratos, observou-se uma grande concentração de clorofila a nos extratos de $L$. digitata e, para os extratos das demais algas, foram identificados como princípios $\mathrm{AO}$ as chamadas substâncias relacionadas com a clorofila $\underline{a}$, tocoferóis, fucoxantina e fosfolipídeos. A fucoxantina não apresentou sinergia

com a vitamina $E$, enquanto a clorofila $\underline{a}$ e suas substâncias relacionadas mostraram um grande efeito sinérgico. A atividade neutralizadora de radicais DPPH e hidroxila $\left({ }^{\circ} \mathrm{OH}\right)$ foi avaliada por Yan et al. (1998) para 27 espécies de algas marinhas, muitas das quais usadas como alimento na Ásia e áreas do Pacífico. O ensaio do DPPH foi feito em atmosfera de nitrogênio, usando dimetilsulfóxido (DMSO) como solvente. Os extratos em metanol foram os que apresentaram melhores resultados nesse ensaio, sendo que 15 espécies do total testado mostraram boa AAO e, dentre essas, 6 espécies destacaram-se: Gelidium amansii, Gloisiphonia capillaris, Polysiphonia urceolata e Rhodomela teres (vermelhas); Sargassum kjellmanianum e Desmarestia viridis (pardas). A atividade neutralizadora de ${ }^{\circ} \mathrm{OH}$ foi avaliada no ensaio da desoxirribose e as espécies $R$. teres e Chorda filium foram as que apresentaram melhores resultados.

Xue et al. (1998) testaram a atividade antioxidante de vários polissacarídeos hidrossolúveis de algas marinhas (alginato, sulfato de alginato, sulfato de propilenoglucolalginato, sulfato de propilenoglucolmanuronato, oligossacarídeo de quitosana, N,O-carboximetilquitosana e hidropropilquitosano)elipossolúveis(hexanoilquitosano e $N$-benzoilhexanoilquitosano). $\mathrm{O}$ ensaio usado para os polissacarídeos hidrossolúveis foi a lipoperoxidação de lipossomas de fosfatidilcolina iniciada pelo cloridrato de 2,2'-azobis(2-amidinopropano) (AZPH), no qual testou-se a propriedade dos diferentes polissacarídeos de inibir o acúmulo de peróxidos da fosfatidilcolina. Para os derivados lipossolúveis, a atividade neutralizadora de radical peróxido foi avaliada pelo monitoramento da concentração do peróxido de metilinoleato, induzido pelo AMVN (2,2'-azobis(2,4-dimetilvaleronitrila). Todos os polissacarídeos testados mostraram AAO, indicando que os derivados de quitina e alginato exercem papel importante no mecanismo antioxidante dos sistemas biológicos.

Yan et al. (1999) avaliaram a AAO frente ao DPPH dos extratos de algumas algas pardas comestíveis no Japão. Os melhores resultados foram encontrados para Undaria pinnatifida, Sargassum fulvellum e Hizikia fusiformis. No fracionamento do extrato dos talos frescos de $H$. fusiformes, a substância com maior AAO foi isolada do extrato em acetônico e identificada como sendo a fucoxatina. Ruberto et al. (Ruberto et al. 2001), avaliaram a AAO de oito espécies do gênero Cystoseira, usando um modelo de sistema micelar, onde se avalia a capacidade da amostra em proteger da peroxidação lipídica o ácido linoleico. Eles fizeram uma correlação entre a AAO e a composição dos extratos em derivados tetrapreniltoluquinóis. Os resultados encontrados mostraram o potencial antioxidante do gênero Cystoseira, diferenciado entre as espécies de acordo com o conteúdo em tetrapreniltoluquinóis dos extratos. Novoa et al. (Novoa et al., 2001), apresentaram 
os resultados da avaliação da atividade inibidora da peroxidação lipídica do extrato aquoso da alga marinha vermelha Bryothamnion triquetrum. Eles determinaram a concentração inibitória média $\left(\mathrm{IC}_{50}\right)$ de $23,3 \mu \mathrm{g}$ para o extrato testado e determinaram para o mesmo um conteúdo em polifenóis de $8,08 \mathrm{mg} / \mathrm{g}$ de extrato liofilizado. Através do fracionamento do extrato puderam identificar a presença dos ácidos trans-cinâmico, -cumárico e ferúlico em concentrações relevantes e associaram, pelo menos em parte, a atividade AO do extrato de $B$. triquetrum à presença dessas substâncias.

Os polissacarídeos sulfatados, de baixo peso molecular da alga parda Laminaria japonica foram avaliados por Xue et al. (2001) quanto a AAO, usando o modelo de oxidação de lipoproteínas de baixa densidade (LDL). Os polissacarídeos testados foram fortemente ativos na proteção da oxidação de LDL induzida por (dicloreto de 2,2'-azobis(2-amidinopropano) (AAPH), mas não para aquela induzida por $\mathrm{Cu}^{2+}$. Rupérez et al. (2002) testaram a AAO dos polissacarídeos de Fucus vesiculosus (alga parda) frente à redução de íons férricos como poder antioxidante no ensaio denominado "ferric reducing ability of plasma" (FRAP), ou seja, a capacidade antioxidante do plasma. Em 2002, Lim et al. (2002) apresentaram um trabalho de avaliação da AAO do extrato de Sargassum siliquastrum, usando o modelo de inibição da oxidação e hemólise de células vermelhas pelo AAPH (dicloreto de 2,2'-azobis(2amidinopropano)), supressão da peroxidação lipídica em homogenato de cérebro de rato e atividade sequestrante de radical $\mathrm{O}_{2} \div$. Eles mostraram que a fração mais ativa era rica em fenólicos, mas não encontraram correlação entre o teor total de fenólicos e AAO. Burritt et al. (2002) fizeram um estudo sobre os danos às membranas e metabolismo da alga vermelha Stictosiphonia arbuscula, imediatamente após reidratação de espécimes dessecadas, e constataram que a capacidade de prevenir ou reduzir a produção de EROs, se deve a um aumento na atividade das enzimas requeridas na regeneração do ascorbato e da glutationa. Esse mecanismo protege a alga de danos celulares causados pela dessecação.

Baseados nos resultados de Foti et al. (1994), Fisch et al. (2003) avaliaram a AAO dos extratos em diclorometano e metanol de Cystoseira crinita, assim como de alguns derivados tetrapreniltoluquinólicos, tetrapreniltoluquinônicos e tripreniltoluquinólicos (Figura 1: estruturas 8-15) isolados e purificados a partir da fração em acetona do extrato metanólico. Foram utilizados os métodos de reação com o radical DPPH, de avaliação de substâncias reativas ao ácido tiobarbitúrico (TBARS), de quimiluminescência e de avaliação da capacidade antioxidante equivalente ao trolox (TEAC). Todas as substâncias testadas apresentaram bom potencial AO, apesar da eficácia variar de acordo com o método usado. Em 2003, Fallarero et al. (2003) avaliaram a capacidade dos extratos aquosos de Bryothamnion triquetrum, uma alga vermelha, e de
Halimeda incrassata, alga verde, coletadas em Havana/ Cuba, em reduzir a formação de EROs intracelular sob condições oxidativas basais, usando uma linhagem de células de hipotálamo de camundongo GT1-7. Também avaliaram o efeito protetor dos extratos destas duas algas contra estímulos químicos $\left(\mathrm{H}_{2} \mathrm{O}_{2}\right.$ e $\left.\mathrm{CH}_{3} \mathrm{HgCl}\right)$ que induzem morte neuronal através de mecanismos envolvendo formação de EROs e depleção de glutationa reduzida (GSH). Ambos os extratos, em concentrações superiores a $0,20 \mathrm{mg} / \mathrm{mL}$, exerceram proteção contra os efeitos deletérios e morte celular induzidos por $\mathrm{H}_{2} \mathrm{O}_{2}$, embora somente o extrato de $H$. incrassata tenha podido prevenir também contra a formação basal de EROs. Quanto aos efeitos deletérios e morte celular, assim como formação de EROs mediados pelo cloreto de metilmercúrio $(\mathrm{MeHgCl})$, os extratos das duas algas mostraram proteção em concentrações maiores que 0,05 $\mathrm{mg} / \mathrm{mL}$.

Nagai e Yukimoto (2003) apresentaram um trabalho sobre o potencial AO de bebidas preparadas com quatro algas comuns na culinária japonesa: Ecklonia cava (trombeta-do-mar), Undaria pinnatifida ("wakame"), Hizikia fusifome ("hizikia") e Ulva pertusa (alface-do-mar). Foram empregados quatro métodos diferentes: avaliação da AAO sobre a peroxidação lipídica do ácido linoleico, atividade neutralizadora do radical $\mathrm{O}_{2}^{-}$no sistema xantina/xantina-oxidase, atividade redutora do radical DPPH e atividade inibidora da oxidação da 2-desoxiribose pelo radical ${ }^{\circ} \mathrm{OH}$ no sistema desoxirribose $/ \mathrm{H}_{2} \mathrm{O}_{2}$. Os autores concluíram que as bebidas têm um bom potencial $\mathrm{AO}$, sendo aquela obtida de trombeta-do-mar mostrou os melhores resultados e aquela obtida de alface-do-mar teve menor atividade. Os mesmos autores também encontraram uma correlação entre o conteúdo de polifenóis e a AAO. Ainda, em 2003, Perez et al. (2003a,b) apresentaram em um congresso internacional os resultados da pesquisa sobre a AAO dos extratos das algas marinhas Laurencia sp., Gelidium sp., Bryothamnion sp. e Gracilaria sp. Os extratos foram avaliados quanto à capacidade $\mathrm{AO}$ frente aos radicais $\mathrm{O}_{2}{ }^{-},{ }^{\circ} \mathrm{OH}$ e radicais gerados sob luz UV. Os autores registraram uma AAO dos extratos comparáveis à de antioxidantes já bem conhecidos como a vitamina $\mathrm{E}$ e a melatonina. Os resultados foram correlacionados com o conteúdo de polifenóis desses extratos. Os mesmos extratos também foram testados quanto à capacidade inibidora da peroxidação lipídica e formação de malondialdeído, através do teste de substâncias reativas ao ácido tiobarbitúrico (TBARS). Kang et al. (2004) fizeram a avaliação da AAO dos extratos de 17 algas marinhas. Três deles, Ulva pertusa, Symphyocladia latiuscula e Ecklonia stolonifera apresentaram boa atividade inibidora da formação de EROs em um modelo usando homogenato de rim de ratos Wistar e diacetato de 2',7'-diclorodiidrofluoresceína. Em seguida, eles testaram as frações solúveis em $n$-hexano, diclorometano, acetato de etila e $n$-butanol do extrato 
<smiles>COc1cc(C)c(O)c(C/C=C(\C)CC(=O)[C@H]2[C@@]3(C)CCCC3(C)[C@@]23CC(O)C(C)(C)O3)c1</smiles>

1

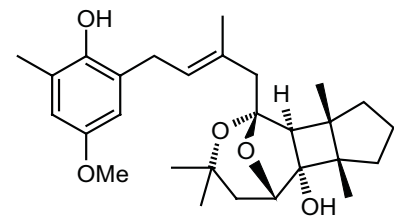

2<smiles>Oc1cc(O)cc(O)c1</smiles>

3 $\mathrm{HO}$ $\mathrm{OH}$<smiles></smiles>

4<smiles>COc1c(O)cc(O)c2c1Oc1cc(O)cc(O)c1O2</smiles>

5<smiles>Oc1cc(O)cc(Oc2c(O)cc(O)c3c2Cc2cc(Oc4c(O)cc(Oc5c(O)cc(O)c6c5Oc5c(O)cc(O)cc5O6)cc4O)ccc2O3)c1</smiles><smiles>[R]C(C)=CCC/C(C)=C/C(=O)C/C(C)=C/Cc1cc(O)cc(C)c1O</smiles>

8

9<smiles>C/C(=C/C(=O)C/C(C)=C/Cc1cc(O)cc(C)c1O)CC/C=C(\C)Cc1cc(C)co1</smiles>

11

Figura 1. Substâncias isoladas de algas marinhas com potencial antioxidante. 
Tabela 1. Avaliação da atividade antioxidante em algas marinhas.

Referencias Conclusões

Fujimoto; Kaneda, 1980, Algas pardas maior atividade AO no teste de estabilidade do éster metilico do óleo

1984

Le Tutour et al., 1990

Cahyana et al., 1992

Foti et al., 1994

Yan et al., 1996

Nomura et al., 1997

Anggadiredja et al., 1997

Matsukawa et al., 1997

Le Tutour, 1998

Yan et al., 1998

Xue et al., 1998

Yan et al., 1999

Ruberto et al., 2001

Novoa et al., 2001

Xue et al., 2001

Rupérez et al., 2002

Lim et al., 2002

Burritt et al., 2002

Fisch et al., 2003

Fallarero et al., 2003

Nagai; Yukimoto, 2003 de girassol

Princípios AO $\rightarrow$ Fosfolipídios e Fucoxantina

AAO dos extratos apolares de algas da costa francesa: isolamento dos princípios $\mathrm{AO}$ e verificação de sinergismo com a vitamina $\mathrm{E}$.

Pirofeofitina $a$ identificada como um dos princípios AO do extrato de Eisenia bicyclis. Atividade $>$ do que $\alpha-\mathrm{TOH}$.

Meroditerpenos de Cystoseira (Pheophyceae): avaliação da AAO frente ao ${ }^{1} \mathrm{O}_{2}, \mathrm{O}_{2}{ }^{-}$ e ROO:

OS $3 \rightarrow$ boa atividade $\mathrm{p} /{ }^{1} \mathrm{O}_{2}$ e nenhuma $\mathrm{p} / \mathrm{O}_{2}{ }^{-}$;

$2 \rightarrow$ boa atividade $\mathrm{p} / \mathrm{ROO}$.

AAO de florotaninos de Sargassum kjellmanianum: boa atividade, 2,6 > BHT

Testou a atividade redutora de carotenóides isolados de algas pardas frente ao DPPH: atividade da fucoxantina melhor em atmosfera de nitrogênio. Os demais $\rightarrow$ $\downarrow$ atividade..

Avaliação da AAO de extratos dos talos frescos e secos de $S$. polycystum: algas secas $\downarrow$ AAO e algas frescas (extrato $\mathrm{MeOH}) \uparrow$ AAO.

AAO de extratos em $\mathrm{H}_{2} \mathrm{O}$ e EtOH de macroalgas frente ao DPPH e lipoxigenase: > Atividade $\mathrm{p} /$ algas pardas nos 2 ensaios

Nenhuma correlação dos 2 testes.

Substâncias relacionadas com clorofila a , tocoferóis, fucoxantina e fosfolipídios como princípios AO presentes em Fucus vesiculosus, F. serratus, e Ascophyllum nodosum.

Avaliação da atividade neutralizante de DPPH e $\bullet \mathrm{OH}$ (ensaio da desoxirribose) dos extratos secos de 27 espécies de macroalgas: $6 \rightarrow$ Boa AAO frente ao DPPH; $2 \rightarrow$

Boa atividade para $\cdot \mathrm{OH}^{\cdot}$

AAO de polissacarídeos de algas: todos os derivados de quitina e alginato mostraram boa AAO.

Avaliação da AAO de algas comestíveis do Japão frente ao DPPH: fucoxantina > atividade (extrato em $\mathrm{Me}_{2} \mathrm{CO}$ ).

AAO do gênero Cystoseira $\rightarrow$ correlação entre AAO e o conteúdo dos extratos em derivados tetrapreniltoluquinóis.

AAO extrato aquoso de Bryothamnion triquetrum sobre a peroxidação lipídica. Presença dos ácidos trans-cinâmico, $\rho$-cumárico e ferúlico.

AAO polissacarídeos sulfatados de $\downarrow$ peso molecular de L. japonica.

Avaliação da atividade antioxidante de polisacarídeos sulfatados de Fucus vesiculosus $\rightarrow$ potencial $\mathrm{p} /$ serem usados como antioxidantes naturais na indústria alimentícia.

AAO de Sargassum siliquastrum, $\neq$ s métodos $\rightarrow$ fenólicos.

Estudo do metabolismo da alga vermelha Stictosiphonia arbuscula: capacidade de prevenir ou reduzir produção de EROs relacionada $\mathrm{c} / \uparrow$ da atividade de enzimas envolvidas na regeneração do ascorbato e GSH.

Avaliação da AAO de meroditerpenóides isolados de Cystoseira crinita, através de diferentes métodos: ensaio do DPPH, de TBARS, TEAC e quimiluminescência. As 14 substâncias testadas $\rightarrow$ AAO relevante.

Atividade neuroprotetora contra efeitos oxidativos deletérios de $\mathrm{H}_{2} \mathrm{O}_{2}$ e $\mathrm{MeHgCl}$, dos extratos aquosos de B. triquetrum e Halimeda incrassata. AAO de B. triquetrum relacionada com $\uparrow$ conteúdo de substâncias fenólicas.

Avaliação da AAO de bebidas preparadas com 4 algas da culinária japonesa, usando 4 métodos diferentes. Correlação do conteúdo de fenóis e AAO. Ecklonia cava > atividade, Ulva pertusa < atividade e as outras duas, Undaria pinnatifida e Hizichia 
fusifomis, atividade intermediária.

Perez et al., 2003a,b

Kang et al., 2004

Ah net al., 2004
Avaliação da AAO de Laurencia sp, Gelidium sp, Bryothamnion sp e Gracilaria sp frente aos radicais $\mathrm{O}_{2}-\bullet \cdot \mathrm{OH}$ e aqueles gerados sob luz UV e ensaio TBARS.

Avaliação da AAO de extratos de 17 algas marinhas e 5 derivados florotanino isolados de Ecklonia stolonifera quanto ao efeito inibitório da geração de EROs: 3 extratos e os 5 derivados florotaninos $\rightarrow$ boa atividade.

Extratos da alga Scytosiphon lomentaria obtidos de material previamente hidrolisados por enzimas carboidrases e proeases $\rightarrow$ AAO frente a radicais $\cdot \mathrm{OH}$,

AO: atividade antioxidante; RL: radical livre; $p \mathrm{O}_{2}$ : pressão parcial de oxigênio; AAO: atividade antioxidante; $\alpha$-TOH: $\alpha$-tocoferol; $\mathrm{O}_{2}^{-}$: ânion superóxido; ROO: radical peróxido; EROs: espécies reativas do oxigênio; TBARS: substâncias reativas ao ácido tiobarbitúrico; TEAC: AAO equivalente ao trolox.

em metanólico de E. stofonifera. A fração em acetato de etila foi a mais potente, sendo isolados 5 derivados fenólicos: o floroglucinol, o eckstolonol, o eckol, o florofucofuroeckol A e o dieckol, (Figura 1, estruturas 3-7), os quais também mostraram atividade muito boa, sendo o eckol e o florofucofuroeckol os mais ativos, inclusive mais ativos que trolox (usado como controle positivo). As algas marinhas, especialmente as algas pardas, são ricas em polissacarídeos como alginatos, fucanos e laminaranos, os quais possuem várias propriedades biológicas, mas poderiam interferir na solubilidade total em água dos extratos de algas. Assim, em 2004, Ahn et al. (2004) apresentaram um trabalho em que prepararam extratos hidrossolúveis de Scytosiphon lomentaria (alga parda) a partir de material previamente hidrolisado por enzimas carboidrases (5 classes) e proteases (5 classes). Esses extratos foram testados quanto a AAO frente aos radicais ${ }^{\circ} \mathrm{OH}$, alquila e $\mathrm{DPPH}$, usando a técnica de ressonância de elétron spin. Todos os 10 extratos obtidos tiveram sua atividade antioxidante similar àquela da vitamina $\mathrm{C}$ frente aos radicais ${ }^{\circ} \mathrm{OH}$ e alquila e menor em relação ao radical DPPH. Os autores sugerem que extratos assim preparados têm a vantagem de melhor solubilidade em água e maior facilidade de processamento.

Os presentes autores acreditam no grande potencial da flora marinha bentônica como fonte de fármacos e insumos farmacêuticos diversificados e, principalmente no que diz respeito às diversas patologias associadas ao estresse oxidativo.

\section{CONCLUSÃO}

Substâncias antioxidantes podem atuar através de mecanismos variados como capturar ou regenerar radicais livres, decompor peróxidos, extinguir $\mathrm{O}_{2}{ }^{1}$, inibir enzimas envolvidas no processo de formação de radicais livres, inibir a cascata de reação dos radicais livres, entre outros. Por essa razão, a capacidade de uma variedade de substâncias antioxidantes em atuar como finalizadores do processo em cadeia das reações radicalares tem sido indiretamente avaliada, através de métodos diversos, usando vários modelos. Para muitos desses métodos, os resultados obtidos dependem do modelo usado e da hidrofilicidade/lipofilicidade da amostra antioxidante testada. Assim, devido às diferenças entre os sistemas de testes empregados para se fazer a avaliação da atividade, recomenda-se o uso de pelo menos dois métodos, dependendo do potencial esperado e da origem da amostra.

As algas marinhas, assim como as plantas superiores, podem ser uma importante fonte de substâncias antioxidantes naturais e, de fato, constam na literatura vários trabalhos de busca por substâncias com atividade antioxidante em algas, porém, no Brasil, apesar da riqueza da nossa flora ficológica marinha, esse campo de pesquisa ainda não foi devidamente explorado.

\section{AGRADECIMENTOS}

Os autores agradecem ao CNPq pelo auxílio à pesquisa e pela bolsa concedida à F.D.R e bolsas de produtividade de V.T.L., R.C.P e M.A.C.K.; à CAPES pela bolsa concedida à F.D.R no programa $\mathrm{PDEE}$ (Programa de Doutorado com Estágio no Exterior) e a HEFCE (The Higher Education Funding Council for England) pelo auxílio à pesquisa.

\section{REFERÊNCIAS}

Ahn CB, Kang DS, Shin TS, Jung BM 2004. Free radical scavenging activity of enzymatic extracts from a Brown seaweed Scytosiphon lomentaria by electron spin resonance spectrometry. Food Res Int 37: 253-258.

Anggadiredja J, Andyam R, Hayati M 1997. Antioxidant activity of Sargassum polycystum (Phaeophyta) and Laurencia obtusa (Rhodophyta) from Seribu Islands. J Appl Phycol 9: 477-479.

Burritt DJ, Larkindale J, Hurd CL2002. Antioxidant metabolism in the intertidal red seaweed Stictosiphonia arbuscula following desiccation. Planta 215: 829-838.

Cahyana AH, Shuto Y, Kinoshita Y 1992. Pyropheophytin a as antioxidative substance from the marine alga, arame (Eisenia bicyclis). Biosc Biotech Bioch 56: 
$1533-1535$

Cahyana AH, Shuto Y, Kinoshita Y 1993. Synergistic antioxidative effects of porphyrin derivatives with $\alpha$-tocopherol and ascorbic acid. Biosci Biotech Bioch 57: 1753-1754.

Fallarero A, Likkanen JJ, Männistö PT, Castañeda O, Vidal A 2003. Effects of aqueous extracts of Halimeda incrassata (Ellis) Lamourox and Bryothamnion triquetrum (S.G.Gmelim) Howe on hydrogen peroxide and methyl mercury-induced oxidative stress in GT1-7 mouse hypothalamic immortalized cells. Phytomedicine 10: 39-47.

Favier AE, Cadet J, Kalyanaraman B, Fontecave M, Pierre JL 1995. Analyses of Free Radicals in Biological Systems. Basel/Switzerland: Birkhauser Verlag. $275 \mathrm{p}$.

Fisch KM, Böhm V, Wright AD, König GM 2003. Antioxidative meroterpenoids from the brown alga Cystoseira crinita. J Nat Prod 66: 968-975.

Fleschin S, Fleschin M, Nita S, Pavel E, Magearu V 2000. Free radicals mediated protein oxidation in biochemistry. Roum Biotech Lett 5: 479-495.

Foti M, Piattelli M, Amico V, Ruberto G 1994. Antioxidant activity of phenolic meroditerpenoids from marine algae. J Photochem Photobio B 26: 159-164.

Fujimoto K, Kaneda T 1980. Screening test for antioxigenic compounds from marine algae and fraction from Eisenia bicyclis and Undaria pinnatifida. Bull Japan Soc Sci Fisheries 46: 1125-1130.

Fujimoto K, Kaneda T 1984. Separtion of antioxygenic (antioxidant) compounds from marine algae. Hydrobiologia 116: 111-113.

Kang HS, Chung JY, Kim JY, Son BW, Jung HA, Choi JS 2004. Inhibitory phlorotannins from the edible brown alga Ecklonia stolonifera on total reactive oxygen species (ROS) generation. Arch Pharm Res 27: 194-198.

Le Tutour B, Benslimane F, Gouleau MP, Gouygou JP, Saldan B, Quemeneur F 1990. Antioxidative activities of algae extracts, synergistic effect with vitamin E. Phytchemistry 29: 3759-3765.

Le Tutour B 1998. Antioxidant and pro-oxidant activities of the brown algae, Laminaria digitata, Himanthalia elongata, Fucus vesiculosus, Fucus serratus and Ascophyllum nodosum. J Appl Phycol 10: 121-129.

Lim SN, Cheung PCK, Ooi VEC, Ang PO 2002. Evaluation of antioxidant activity of extracts from a brown seaweed, Sargassum siliquastrum. J Agric Food Chem 50: 3862-3866.

Matkovics A 2003. An overview of free radical research. Acta Biologica Szegediensis 47: 93-97.

Matsukawa R, Dubinsky Z, Kishimoto E, Masaki K, Masuda Y, Takeuchi T, Chihaara M, Yamamoto Y, Niki E, Karube I 1997. A comparison of screening methods for antioxidant activity in seaweeds. $J$ Appl Phycol 9: 29-35

Nagai T, Yukimoto $\mathrm{T}$ 2003. Preparation and functional properties of beverages made from sea algae Food
Chem 81: 327-332.

Nordberg J, Arnér SJ 2001. Reactive oxygen species, antioxidants, and the mammalian thioredoxin system. Free Radical Biol Med 31: 1287-1312.

Novoa AV, Motidome M, Mancini-Filho J, Linares AF, Tanae MM, Torres LMB, Lapa AJ. 2001. Actividad antioxidant y ácidos fenólicos del alga marina Bryothamnion triquetrum (S.G.gmelim) Howe. Braz J Pharm Sci 37: 373-382.

Olszewer E 1995. Radicais Livres em Medicina $2^{\text {a }}$ ed. São Paulo: Fundo Editorial Byk, 204 p.

Olszewer E, Carter JP 1988. EDTA Chelation therapy in chronic degenerative disease. Medical Hypotheses 27: 41-49.

Perez E, Rodríguez-Mlaver A, Padilla N, Medina-Ramirez G, Laenna E 2003a. Antioxidant capacity of seaweed extracts on superoxide anion, hydroxyl radical and radicals generated by UV-light. Free Rad Biol Med 35(Suppl. 1): 117.

Perez E, Rodríguez-Mlaver A, Padilla N, Medina-Ramirez G, Laenna E 2003b. Antioxidant capacity of seaweed extracts during lipid peroxidation wister rat homogenates. Free Rad Biol Med 35(Suppl. 1): 121

Pietta PG 2000. Flavonoids as antioxidants. J Nat Prod 63: 1035-1042.

Rice-Evans CA, Miller NJ, Paganga G 1996. Structureantioxidant activity relationships of flavonoids and phenolic acids. Free Rad Biol Med 20: 933-956.

Rover Júnior L, Höehr NF, Vellasco AP 2001. Sistema antioxidante envolvendo o ciclo metabólico da glutationa associado a métodos eletroanalíticos na avaliação do estressse oxidativo. Quim Nova 24: 112-119.

Ruberto G, Baratta MT, Biondi DM, Amico V 2001. Antioxidant activity of extracts of the marine algal genus Cystoseira in a micellar model system. J Appl Phycol 13: 403-407.

Rupérez P, Ahrazem O, Leal JA 2002. Potential antioxidant capacity of sulfated polysaccharides frrom the edible marine Brown seaweed Fucus vesiculosus. J Agr Food Chem 50: 840-845.

Sies H 1993. Strategies of antioxidant defense. Eur J Biochem 215: 213-219.

Tiwari AK 2001. Imbalance in antioxidant defense and human diseases: Multiple approach of natural antioxidants therapy. Curr Sci 81: 1179-1187.

Tully S 1999. EDTA chelation therapy. Altern Integr Med Soc I: 7.

Xue C-H, Yu G, Hirata T, Terao J, Lin H 1998. Antioxidative activities of several marine polysaccharides evaluated in a phosphatidylcholine-liposomal suspension and organic solventes. Biosci Biotech Bioch 62: 206-209.

Xue C-H, Fang Y, Lin H, Chen L, Li Z-J, Deng D, Lu C-X 2001. Chemical characters and antioxidative properties of sulfated polysaccharides from Laminaria japonica. $J$ Appl Phycol 13: 67-70. 
Yan X, Xiancui L, Chengxu Z, Xiao F 1996. Prevention of fish oil rancidity by phlorotannins from Sargassum kjellmanianum. J Appl Phycol 8: 201-203.

Yan X Nagata T, Fan X 1998. Antioxidative activities in some common seaweeds. Plant Food Hum Nutr 52: 253-262.

Yan X, Chuda Y, Suzuki M, Nagata T 1999. Fucoxanthin as the major antioxidant in Hijikia fusiformis, a common edible seaweed. Biosci Biotech Bioch 63: 605-607. 